\title{
Unusual thermoelectric behavior of packed crystalline granular metals
}

\author{
M. Ausloos ${ }^{\text {a) }}$ \\ SUPRATECS, Institute of Physics B5, University of Liège, B-4000 Liège, Belgium
}

M. Pȩkala

Department of Chemistry, University of Warsaw, Al. Zwirki i Wigury 101, PL-02-089 Warsaw, Poland

J. Latuch

Department of Materials Science and Engineering, Warsaw University of Technology, ul. Woloska 141, PL-02-507 Warsaw, Poland

J. Mucha

Institute of Low Temperature and Structure Research, Polish Academy of Sciences, P.O. Box 1410, 50-950

Wroclaw, Poland

Ph. Vanderbemden

SUPRATECS, Institut d'Electricité Montefiore B28, University of Liège, B-4000 Liège, Belgium

\author{
B. Vertruyen and R. Cloots \\ SUPRATECS, Department of Chemistry B6, University of Liège, B-4000 Liège, Belgium
}

(Received 5 May 2004; accepted 29 August 2004)

\begin{abstract}
Loosely packed granular materials are intensively studied nowadays. Electrical and thermal transport properties should reflect the granular structure, as well as intrinsic properties. We have compacted crystalline CaAl-based metallic grains and studied the electrical resistivity and the thermoelectric power as a function of temperature $(T)$ from 15 to $300 \mathrm{~K}$. Both properties show three regimes as a function of temperature. It should be pointed out: (i) The electrical resistivity continuously decreases between 15 and $235 \mathrm{~K}$, (ii) with various dependencies, e.g., $\simeq T^{-3 / 4}$ at low $T$, while (iii) the thermoelectric power (TEP) is positive, (iv) shows a bump near $60 \mathrm{~K}$, and (v) presents a rather unusual square root of temperature dependence at low temperature. It is argued that these three regimes indicate a competition between geometric and thermal processes-for which a theory seems to be missing in the case of TEP. The microchemical analysis results are also reported, indicating a complex microstructure inherent to the phase diagram peritectic intricacies of this binary alloy. (C) 2004 American Institute of Physics. [DOI: 10.1063/1.1808248]
\end{abstract}

\section{INTRODUCTION}

There are different types of dense granular materials, i.e., those in which grains are dispersed in a solid matrix in contrast to loosely packed granular materials. The former class is necessarily a random mixture of two or more phases with different $I-V$ characteristics. It has been much investigated from a fundamental point of view along the lines of percolation ideas, for example, to search in the case of superconducting embedded networks ${ }^{1}$ for the so-called critical concentration, whence percolation critical exponents, etc. ${ }^{2,3}$ In the second class, the mixture can be a powder of even a single chemical type of grains. This class, containing wet or dry mixtures, is of interest for the technological questions they raise, like for powder, sand, or pill packings. ${ }^{4,5}$ In between, stem the loosely packed colloid deposits and polymerlike composites. 6,7

Depending on the volume fraction they occupy, powders can be more or less dense. They can be distinguished from a mechanical or optical point of view, i.e., according to the surface deformation upon loading ${ }^{8}$ or to the modification of the absorbing spectrum ${ }^{9-12}$ (recall "invisible" U2 planes). In the case of electrically insulating grains, recall that structural

${ }^{\text {a)} E l e c t r o n i c ~ m a i l: ~ m a r c e l . a u s l o o s @ u l g . a c . b e ~}$ properties, e.g., of sand or rice piles, are much studied. ${ }^{4,5}$ The thermal properties, like the heat capacity and the thermal conductivity, e.g., of sand, are of interest as well.

The electrical properties of such systems are also of interest when the grains are metalliclike. ${ }^{13}$ It is thought that there is a strong modification of the interface when an electric current is imposed-leading to a strong modification of the oxide layers formed at the grain surfaces ${ }^{14-16}$ - a modification going even up to welding. This the Calzecchi-Onesti transition ${ }^{17}$ leading to sharp transitions in the $I-V$ characteristics when the current reaches a certain value at which the resistance falls several orders of magnitude. Microsolderings between grains may occur before a Calzecchi-Onesti transition, ${ }^{18}$ just like when nucleation sites are initiated by high-concentration gradients at a first-order phase transition. Those solderings are nonequilibrium or irreversible processes, thereby, being the fundamental cause for the hysteretic behavior found in $I-V$ curves of granular packing. In the case of not too good conductors, this leads to the study of hot spots, equally detrimental in superconducting devices. ${ }^{19}$ There are very few papers dealing with the temperature dependence of such electrically conductive- (loosely) packed materials - none to our knowledge about the thermoelectric power (TEP) temperature dependence. 
In huge contrast, studies of the electrical resistance $R(T)$ of inhomogeneous composites have been of continuing interest, $^{20,21}$ with much emphasis on low-temperature phenomena because of superconductivity ${ }^{22-24}$ and tunneling effects; e.g., see Refs. 25-28 and references therein. Interestingly, the thermoelectric power $Q(T)$ has been measured in ceramics, like alumina or SiGe alloys ${ }^{29,30}$ and composite superconductors. It is fair to mention for completeness a few papers on glassy systems ${ }^{31-35}$ _although these are not truly packed systems, but are nevertheless close to...close-packed configurations.

Except for insulating glasses, most composite systems are often made of electrically conducting grains embedded in an insulator matrix, thus behave like a disordered system in the (usually) localized regime. Transport properties depend whether or not the system is above a percolation transition or not; they are usually dominated by carrier hopping between grains, i.e., an intergrain process. Thus, they display certain features of the variable-range-hopping (VRH) phenomenon type observed in doped semiconductors, in fact. In particular, the electrical resistivity of such systems has been observed to obey a so-called stretched exponential dependence

$$
\rho(T)=\rho_{0} e^{\left[\left(T_{0} / T\right)^{p}\right]},
$$

where $T$ is the temperature, $T_{0}$ is a characteristic temperature, and $p=1 /(d+1)$ in terms of the dimensionality $d$ of the system. $^{36-38}$

Yet, it seems obvious that from both structural and chemical points of view, the dense but loosely packed granular systems should be quite different from doped materials. In a (loosely or not) packed granular system, the metal (or conducting entity) occupies a finite volume in space, and hopping from a grain to a (nearest neighbor) grain is likely to occur through rather wide and often inhomogeneous insulating barriers with finite thickness distributed over a sort of percolating network. ${ }^{2,14,15,39,40}$

In contrast, in the case of a doped material, hopping between two impurity sites goes through only a narrow size insulating homogeneous barrier.

Therefore, some debate may arise on whether the VRH model of doped materials can be applied to granular(loosely or densely) compacted metals - the more so for the TEP or Seebeck coefficient, which is a much more elusive quantity $^{41-43}$ and does not seem to be found when browsing through the VRH literature, whence the main motivation behind our studies.

Such electrical and thermal transport properties may be depending on many conditions, like various types of grainsize distributions. This opens up a huge set of problems, which cannot be all tackled here. The effective conductivity and thermoelectric power in such composite systems can be enhanced or reduced-the more so as a function of temperature when there are competitive processes. At this stage, it should be pointed out as references based on macroscopic points of view, with considerations based on effective properties depending on the type of packing as in Refs. 44-53 to quote a few about the (effective-medium approximation) electrical conductivity, while in the case of the thermoelectric power, see Refs. 54-57.
In conclusion of the above, it is clear that some attention should be focused on the possible competition between geometric and thermal influence with some subsequent interpretation based on electronic fundamentals.

A simple binary alloy $\mathrm{Ca}_{x} \mathrm{Al}_{y}$ was selected as an interestingly representative system. The phase diagram is partially known since 1928 according to Ref. 58. It was studied when attempting to make crystals from systems, which were initially glassy. Previous investigations did not report any crystalline phase characteristics, nor a fortiori temperaturedependent properties of crystalline-packed grains.

As described in Sec. II, an original route has been used for the synthesis of $\mathrm{CaAl}_{2}$; we have obtained tiny crystals $(0.5 \mathrm{~mm})^{3}$. After compacting the granular material, we have measured the electrical resistivity $(\rho)$, thermoelectric power $(Q)$, and magnetic properties as a function of temperature (Sec. III) from 40 to $300 \mathrm{~K}$ or so. The temperature dependencies of $\rho$ and $Q$ are found to be unusual. The magneticsusceptibility behavior is paramagnetic (Sec. III). The finding interpretation relies on temperature-dependent percolation network features and stresses intergrain and intragrain differences occurring in such granular-packed materials. Band-structure considerations from Huang and Corbett ${ }^{59}$ on related $\mathrm{Ca}_{x} \mathrm{Al}_{y}$, with various $x$ and $y$, are taken into account in view of interpreting our findings (Sec. IV). The last section (Sec. IV) serves as much as a place for conclusion as for raising questions and suggesting further investigations. The chemical characteristics of the grains are found in the Appendix.

\section{SAMPLE SYNTHESIS AND CHEMICAL CHARACTERISTICS}

Several $\mathrm{Ca}_{x} \mathrm{Al}_{y}$ phases and stoichiometric compounds are known. ${ }^{58}$ Recently, Huang and Corbett ${ }^{59}$ synthesized several of these and characterized them, e.g., $\mathrm{Ca}_{13} \mathrm{Al}_{14}$ and $\mathrm{Ca}_{8} \mathrm{Al}_{13}$, from an electrical resistivity point of view, including some calculation and discussion of the band structure of both compounds. Both materials were found to be good electrical conductors, $\rho_{R T} \simeq 55$ and $\simeq 60 \mu \Omega \mathrm{cm}$, respectively (with paramagnetic Pauli properties). Huang and Corbett ${ }^{59}$ conclude on a very similar density of states near the Fermi level.

Our choice of the $x$ and $y$ values for our study has been influenced by observing the similarity of the $\mathrm{Ca}_{x} \mathrm{Al}_{y}$ phase diagram with that of $\mathrm{BiSrCaCuO}$ or $\mathrm{YBaCuO}$ superconducting ceramics, i.e., a peritectic point near $x=1$ and $y=2$, a compound not studied in the crystalline phase to our knowledge. It is expected that the phase diagram analogy could be of interest for interpreting features, taking into account the grain intrinsic property, if necessary.

Master alloys were prepared in an arc furnace under spectrally pure argon atmosphere with a $0.2-\mathrm{MPa}$ overpressure. Titanium getter was used in order to eliminate traces of oxygen originating from calcium. The purity of starting metals was $5 \mathrm{~N}$ and $3 \mathrm{~N}$ for $\mathrm{Al}$ and $\mathrm{Ca}$, respectively. Samples were homogenized for $15 \mathrm{~min}$ at $1420 \mathrm{~K}$.

The sample is made of very tiny black but shining grains. Even though no size dispersion distribution was 


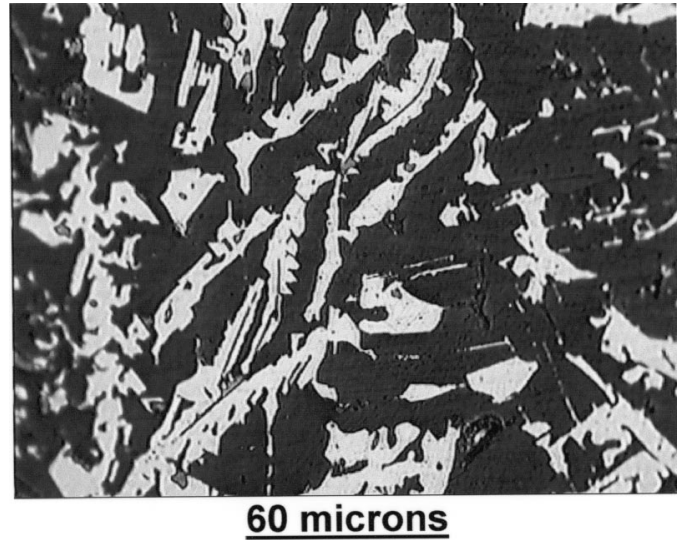

FIG. 1. Polarized light microscopy analysis of a grain of the investigated system, showing $\mathrm{CaAl}_{2}$ dendrites, in a $\mathrm{Ca}_{13} \mathrm{Al}_{14}-\mathrm{Ca}_{8} \mathrm{Al}_{3}$ matrix; the size of the photography diagonal is about $100 \mu \mathrm{m}$.

looked for, the size of the grains seems to be rather uniform, from visual observation, and in the order of $(0.5 \mathrm{~mm})^{3}$. The samples are very brittle even after strong packing in a classical press. After metallographic treatment of the sample surfaces, a grain content is seen through a high-resolution polarized light microscope (Fig. 1) to be made of dendrites embedded in a matrix, the dendrite long size roughly ranging between 10 and $50 \mu \mathrm{m}$. Alas, it has been impossible to extract the needles from the matrix. The dendrites are $\mathrm{Al}$ rich and made of $\mathrm{CaAl}_{2}$, while the matrix is $\mathrm{Al}$ poor and has a composition close to $\mathrm{Ca}_{0.6} \mathrm{Al}_{0.4}$. The chemical characteristics and a comment on the grow process, whence the grain structural (unavoidable) inhomogeneity, can be found in the Appendix. It is shown that the grains are crystalline.

\section{MEASUREMENTS}

\section{A. Electrical resistance}

The four-probe method was used for precise measurements of the electrical resistance of the samples. A dc up to $10 \mathrm{~mA}$ was injected as 1-s pulses with successively reversed direction in order to avoid the Joule and Peltier effects ${ }^{60,61}$ and ageing. ${ }^{14}$ The electrical resistivity was calculated from the voltage drop and the sample dimensions, i.e., $7 \times 7$ $\times 10 \mathrm{~mm}^{3}$. The temperature was varied between 20 and

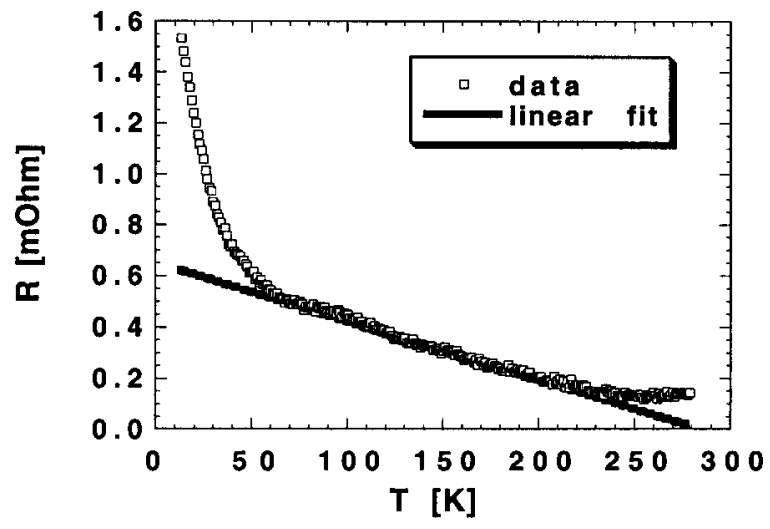

FIG. 2. Electrical resistance vs temperature of a polycrystalline dense packed sample based on $\mathrm{CaAl}_{2}$; the sample dimensions are $7 \times 7$ $\times 10 \mathrm{~mm}^{3}$, leading to a resistivity at room temperature $\simeq 100 \mu \Omega \mathrm{cm}$.

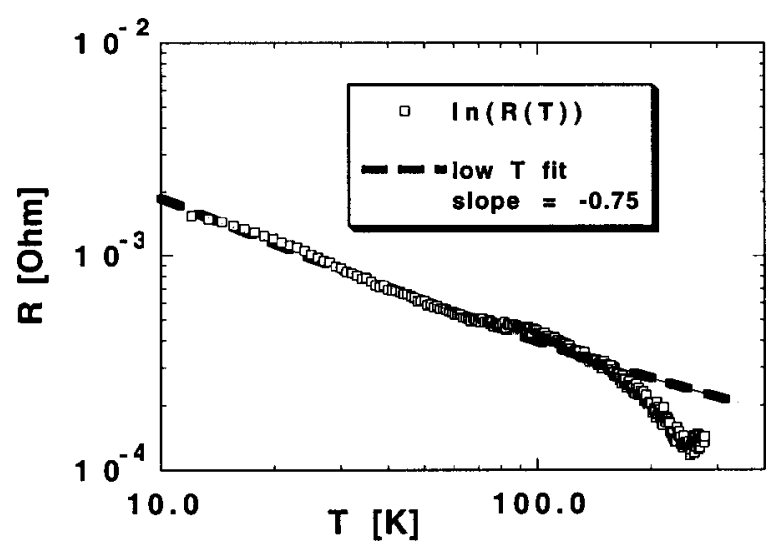

FIG. 3. Same as on Fig. 2, on a $\log -\log$ plot, with fit to a line with slope $=-3 / 4$ at low temperature.

$300 \mathrm{~K}$ in a closed cycle refrigerator with stabilization by a LS340 controller with accuracy of $0.01 \mathrm{~K}$. See other details elsewhere. ${ }^{62}$

The electrical resistance versus temperature variation $R(T)$ measured in a broad temperature range is shown in Fig. 2 and present three regimes. Different unusual features can be observed. First of all, $R$ decreases with increasing $T$ from $20 \mathrm{~K}$ down to $235 \mathrm{~K}$, in particular, there is a quasilinear decay between 70 and $235 \mathrm{~K}$. On a log-log plot (Fig. 3), we observe that $R$ remarkably well behaves like $\sim T^{-3 / 4}$ for $15 \mathrm{~K}<T<70 \mathrm{~K} . R(T)$ seems to remain rather constant for higher $T$ or slightly increasing above $235 \mathrm{~K}$. The electrical resistivity is about $\rho=100 \mu \Omega \mathrm{cm}$ at room temperature.

\section{B. Thermoelectric power}

The TEP has been measured following the method described in Ref. 61. The imposed temperature gradient is about $1 \mathrm{~K} / \mathrm{cm}$. The results are shown in Fig. 4 as $Q$ versus $T$. $Q(T)$ is positive in the investigated temperature range and about $20 \mu \mathrm{V} / \mathrm{K}$ at $77 \mathrm{~K}$ and $40 \mu \mathrm{V} / \mathrm{K}$ at room temperature, respectively-values similar to those of poorly metallic systems. The data presents a bump at ca. $60 \mathrm{~K}$ followed by a minimum at $100 \mathrm{~K}$ and a rapid rise thereafter $\left(\sim 0.12 \mu \mathrm{V} / \mathrm{K}^{2}\right)$. In Fig. 5, a $\ln (Q / T)$ versus $\ln (T)$ plot is shown in which a triple regime is well observed. Below $60 \mathrm{~K}, Q(T) / T \simeq 20 / \sqrt{T}$, thus $Q(T) \simeq 20.0 T^{0.5}$. In the inter-

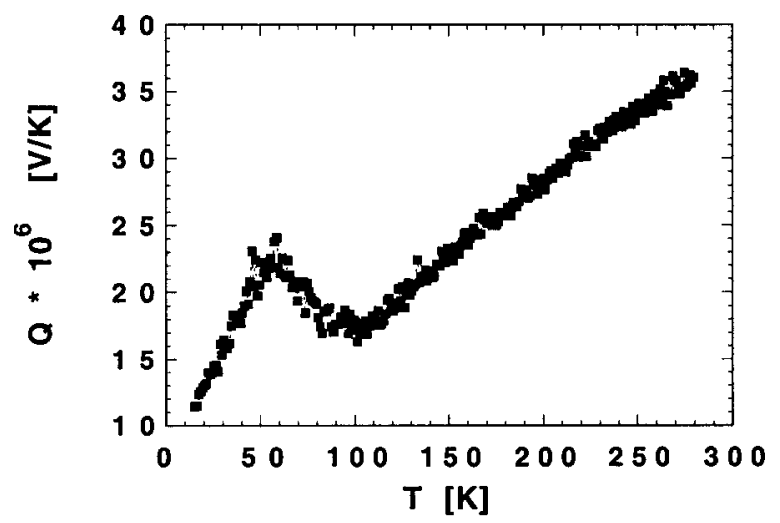

FIG. 4. Thermoelectric power $Q(T)$ vs temperature $T$ of a polycrystalline dense packed sample showing an $N$-shape behavior. 


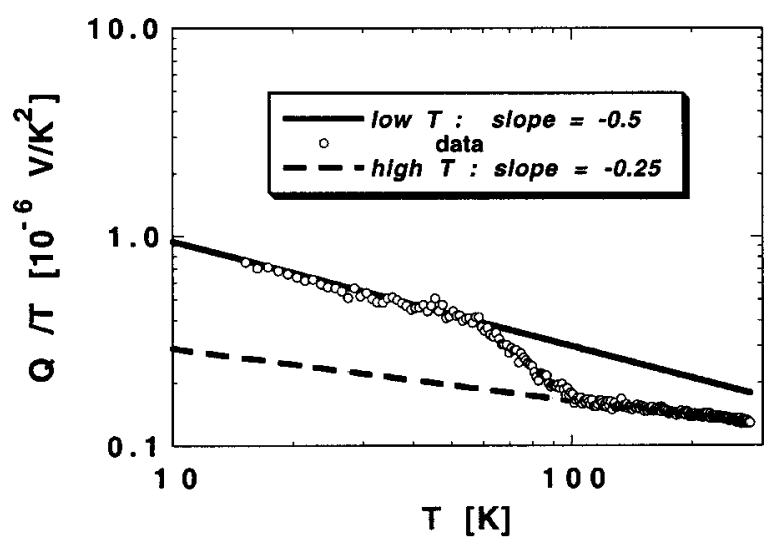

FIG. 5. Same as on Fig. 4 but for $Q(T) / T$ vs temperature $T$ on a $\log -\log$ plot, with fits at low and high temperature.

mediary regime, the decay is very regular till $100 \mathrm{~K}$. Above $100 \mathrm{~K}$, the slope is equal to -0.25 on such a log-log plot, indicating that $Q(T)$ behaves like $T^{3 / 4}$, thus not quite linearly as it should be usual for metallic systems at high $T$.

\section{Magnetic susceptibility}

dc magnetic measurements at several temperatures $(20-300 \mathrm{~K})$ and magnetic fields (up to $1 \mathrm{~T}$ ) were also performed in a Quantum Design Physical Property Measurement System, when it worked, using an extraction method.

The response of the material is that of a paramagnetic one over the whole temperature range investigated, with a susceptibility in the order of $8.610^{-6}$ (SI units) at $T$ $=300 \mathrm{~K}$. It is quasi-independent of temperature; the susceptibility decreases by a factor of 3 between 50 and $300 \mathrm{~K}$ in a field of $1 \mathrm{~T}$, but this is not displayed here.

\section{DISCUSSION}

The discussion is essentially based on the microstructure optically and microscopically observed, i.e., a packed mixture of (apparently heterogeneous, see Appendix) tiny grains, together with the unusual temperature dependence of the electrothermal property for stressing the features and emphasizing fundamental considerations.

First, let it be observed that even though each grain is surely multiphasic (see Appendix), both phases, according to Huang and Corbett, ${ }^{59}$ have equivalent electrical (metallic) properties. Therefore, for discussing the electrical resistivity, at least, we can safely assume that each grain is monophasic and metallic. If the grains were made of insulating components in a not well conducting matrix, thus having a peculiar heterogeneity as found in metal-insulator composites, one might argue that charging effects on inner surfaces might complicate the analysis. This does not seem to be the case here, whence giving some validity for effective-medium theory considerations in describing each grain.

One might next wonder whether the sample network structure could be much modified as a function of temperature, leading to a temperature-dependent (macrostructural) transition. Even though we cannot entirely disregard such effects due to grain thermal expansions, we assume that the granular network structure, whence the number of contacts

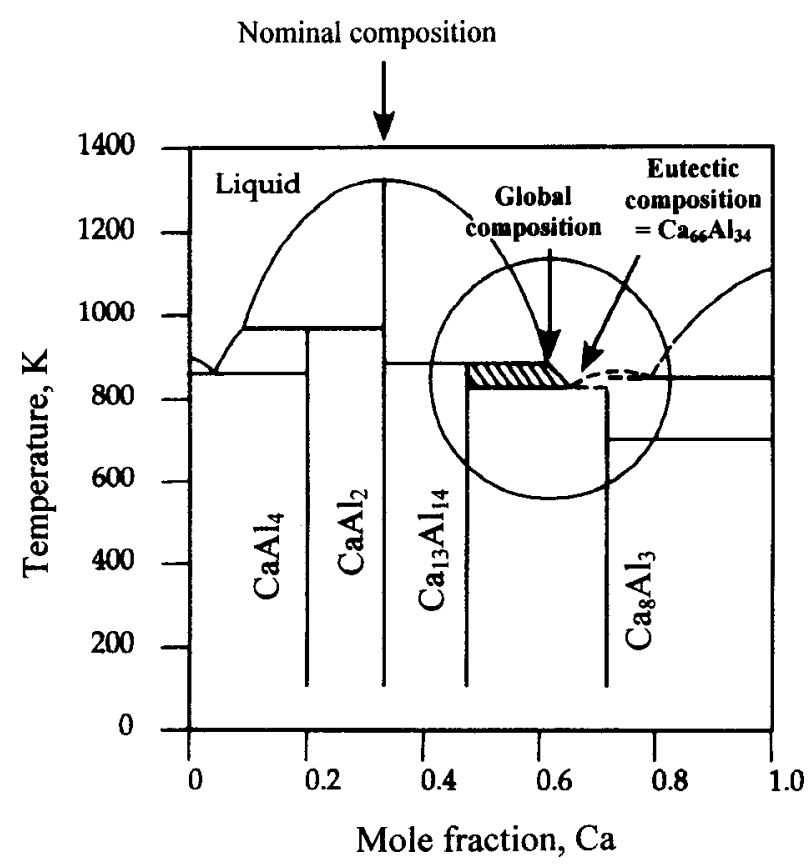

FIG. 6. Phase diagram of the binary system CaAl, extracted from Ref. 76 .

should be rather stable. However, the intergrain connections could be temperature dependent. Therefore, the electrical conduction process should be only resulting from an intergrain hopping process and an intragrain (metalliclike) conductivity. Both having a different temperature dependence. Again, an effective-medium theory should be valid.

\section{A. Electrical resistance}

Various mechanisms are invoked when explaining a decreasing $R(T)$ behavior: (i) an Arrhenius excitation, i.e., an exponential decrease corresponding to a thermal barrier leakage when the temperature increases; (ii) a Mott, so-called VRH mechanism, ${ }^{36,37}$ or (iii) a Shklovskii-Efros (SE) effect, ${ }^{38,63}$ i.e., a small Coulomb gap opens up at the Fermi surface as a result of Coulomb interactions between the quasiparticle carriers, thereby modifying the Mott VRH result. In these cases, the electrical resistivity can be expressed as the stretched exponential, Eq. (1), where $p=1 / 4$ or $1 / 2$ for the Mott or SE type conductivity; $T_{0}$ characterizes the variable-range-hopping energy scale. The classical Arrhenius law corresponds to $p=1$. Plots of $\log \rho$ versus $T^{-1 / 2}$ or $T^{-1 / 4}$ look so far from any straight line that they are not displayed; they do not indicate any (relatively extended) linear region, which could convince us of arguing in favor of a Mott or SE mechanism in some range. In fact, it is found from Fig. 3 that below $70 \mathrm{~K}$,

$$
\rho(T)=\hat{\rho}_{0}\left(\frac{T}{77}\right)^{-3 / 4},
$$

with $\hat{\rho}_{0}=25 \mu \Omega \mathrm{cm}$ when $T$ is measured in Kelvin.

Unusual fractional exponents are often interpreted in other condensed matter research through fractal considerations for the underlying (geometrical) network supporting the elementary excitations of interest ${ }^{64}$-themselves having a possible temperature dependence (which scales like their in- 


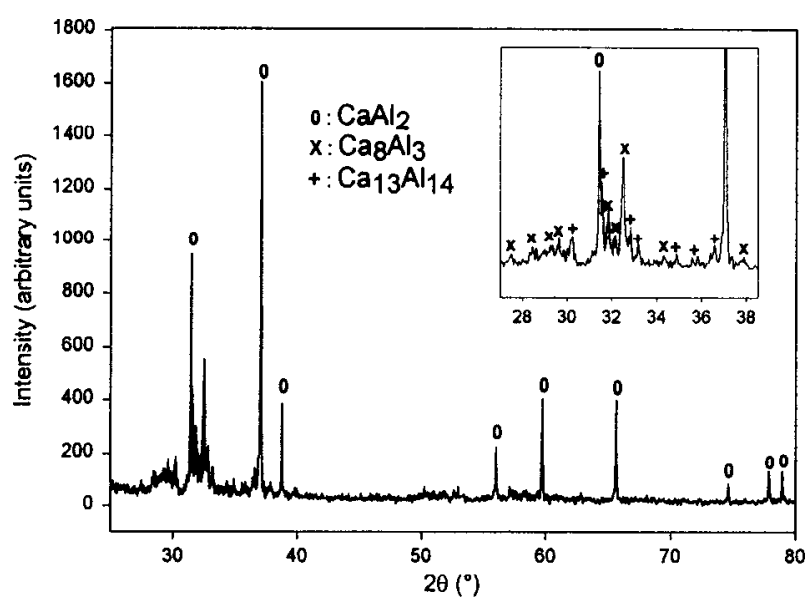

FIG. 7. X-ray diagram of a dense as synthesized CaAl system; the inset points out to peaks belonging to the so-called matrix phase.

trinsic interaction energy). Recall here, as an analogy, the case of magnetic disordered systems, characterized by some exchange energy $J$ (and some directly related) critical temperature $T_{c}$, on a (random) network, characterized by some fractal dimension. The best description of those systems is in fact $^{65}$ through two different coherence lengths, $\xi_{g}$ and $\xi_{T}$, one (usually temperature independent) for the network and one (strongly temperature dependent) for the magnetic structure.

The present electrical conduction process with the temperature behavior of $\rho(T)$, i.e., the $T^{-3 / 4}$ law is best interpreted in terms of a similar argument covering the whole temperature range. In particular, the power-law $\left(\sim T^{-0.75}\right)$ resistance decrease can be thought to result from a thermal effect, on a resistive (geometrically disordered) backbone, not changing with temperature in this low $T$ range. Indeed, in presence of such a geometric disorder, the charge-carrier mean free path $\ell$ can be considered in the usual linear superposition approximation as ${ }^{41}$

$$
\frac{1}{\ell}=\frac{1}{\ell_{T}}+\frac{1}{\ell_{g}},
$$

where $\ell_{T}$ and $\ell_{g}$ are the mean free path of carriers, respectively, due to (grain-size limited) thermal effects and intrinsic geometric disorder. Moreover, in this less conducting, i.e., low-temperature regime, Orbach description of random media excitations ${ }^{64}$ can be used to interpret the exponent $3 / 4$ as the signature of the percolation network for the hopping charge carriers, whence finding an (very reasonable) effective (fractal-like ${ }^{64,65}$ ) dimensionality $\widetilde{\delta}=9 / 4$ of the three $d i$ mensional, since $\widetilde{\delta}>2$, backbone. ${ }^{66}$

The $60-70 \mathrm{~K}$ break (or crossover) indicates the energy range at which the thermal process takes over on the geometric disorder. From a microscopic point of view, this temperature value allows us to estimate the (average) electrical carrier energy distance between the Fermi level and the bottom (observe that $Q(T)>0$, in Fig. 4) of the conduction band. This hopping (or barrier) energy, being overcome at such a temperature, leads to a more easy conduction process, whence a weaker $\rho$. Moreover, above such a $T$, in the socalled intermediate temperature range, the $R(T)$ linear decay reminds us of the behavior found in heavy fermion systems.
Indeed, an electrical resistivity decreasing with temperature, as in Fig. 5, is also observed in heavy fermion materials. ${ }^{67-69}$ Such a feature is associated to a sharp density of states (DOS) near the Fermi level and carrier (de)localization. ${ }^{70}$

Finally, at higher temperature, the smooth decay of $R(T)$ with increasing $T$ can be attributed to the Fermi-Dirac function behavior increasing width as $T$ increases and further charge-carrier delocalization. ${ }^{70}$ The electron (or rather hole, here) band mass contains a more important imaginary contribution as a function of temperature, and the charge-carrier wave function spreads out. The intergrain thermally activated charge (ballistic) hopping process becomes more pronounced at high temperature and competes with ( $p$-like) metallic conduction in the grains. Recall a similar type of mechanism due to (dense) granular structure in mixed superconductors ${ }^{1,27,71}$ like Al-Ge mixture, at "not very low temperatures"72 where the $R(T)$ decay is like $T^{-0.117}$, an exponent similar but quite different from 3/4. Assuming that all (weak) localization effects are suppressed by the temperature, the occurrence of a phase transition in $R(T)$ exists depending on the tunneling conductance $g$ and the phase coherence on both sides of the tunneling junction. For such systems, at small $g$, the conductivity exponentially decays with temperature, but has a logarithmic temperature dependence at large $g$. This (effective) conductance picture can be used analogously here, since the intergrain barrier conductance $g$ likely increases with temperature. However, the temperature dependencies found here above are quite different from those in the granular metals discussed by e.g., Efetov and Tschersich. ${ }^{27}$ In fact, in view of the different temperature dependencies, we prefer to consider that the effects are due to intrinsic density of states rather than mobility constraints at not too low temperature.

\section{B. Thermoelectric power}

Turning to the TEP data, we have already argued elsewhere $^{73}$ that $Q$ should be considered as a transport coefficient and not assimilated only to the change in entropy or density of states. ${ }^{41}$ Nevertheless, the above recalled considerations on mean-free-path selection for the electrical charge-carrier scattering processes are also very useful in order to interpret the $Q(T)$ behavior at low $T$. As in an effective-medium approximation, the behavior of $Q(T)$ depends on the dominant scattering process. Notice that the concept of a percolation network made of tunneling barriers with a distribution of temperature gradients is not usual nor easily simulated for TEP. However, see some similar consideration by Danilov et al. in Ref. 66.

Let us also recall that a TEP measurement implies no external electrical current. Whence, it is unlikely that some "barrier ageing" or "not spots" take place in the temperature range of interest through a Calzecchi-Onesti-like transition. ${ }^{17}$ Moreover, local charging can also be neglected, in view of the metallic nature of the grains-though local hot spots might exist. To our knowledge, they have not been observed in such configurations. Thus, within the above line of thought, both increase in charge and heat transport as a function of temperature, and the large thermoelectric effect at room temperature can also be understood to result from a 


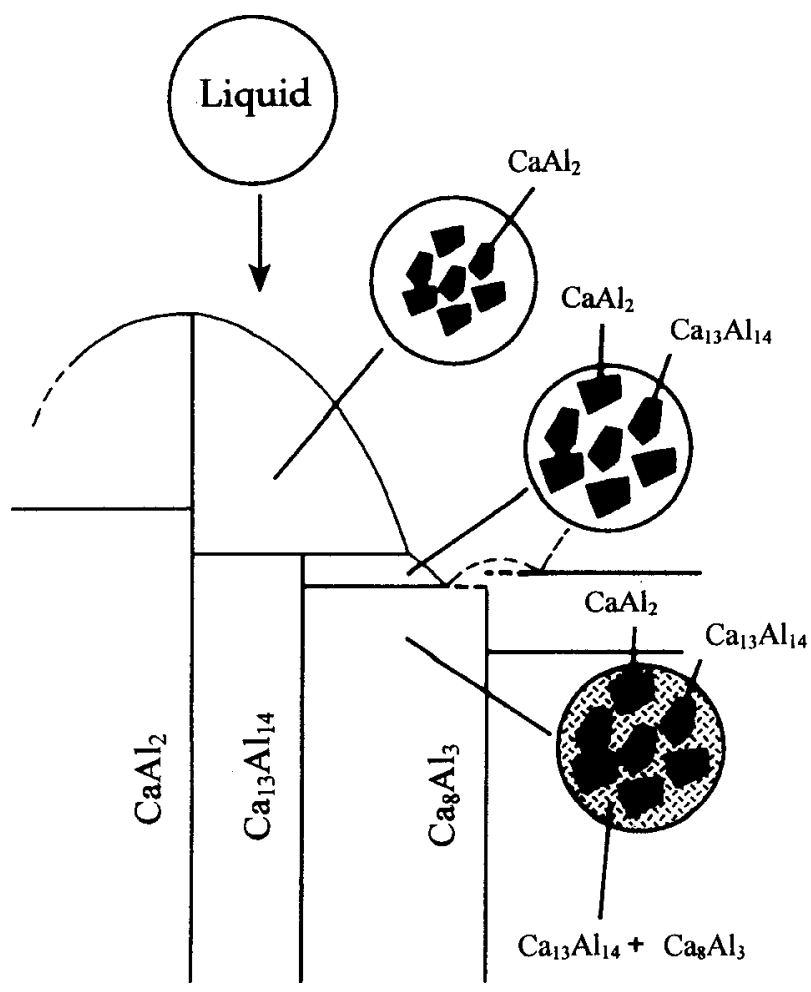

FIG. 8. Sketch of the observed microstructures in relation to the phase diagram of the binary compound $\mathrm{CaAl}$ with emphasis on the peritectic temperature region.

delocalization process on the complex barrier network, with two competing characteristic mean free paths, and a weakening of the contact TEP due to Fermi-surface widening with temperature. There is, to our knowledge, no such a theory for TEP.

To be fair, one might also argue on whether the $N$ shape of $Q(T)$ is not due to a phonon drag. Our argument to dismiss phonon drag, in favor of competing carrier localization lengths, stems in the fact that phonon drag is usually associated to long wavelength phonons and are thus sensitive mainly to boundary scattering. In view of the grain size and granular packing, it could be the case here. However, phonon drag description leads to a predicted behavior like $T^{3}$ for metallic systems - this is not found here at all. As an argument, recall that TEP of compacted small size $(1 \mathrm{~mm})$ homogeneous Ge grains has been previously studied at low temperature, without any convincing evidence that the behavior was due to phonon drag. ${ }^{74-76}$ In fact, a $Q \sim T^{-9 / 2}$ should be expected, ${ }^{77}$ a very different behavior from what is observed here. Whence, it can be discounted that the peak in TEP is due to phonon drag processes. We thus propose that the features are due to an intrinsically competitive (geometric-thermal) dissipation mechanism, involving intraand intergrain parameters. Both a change in DOS and/or electronic heat conduction scattering should be taken into account in theoretical work. ${ }^{41}$

\section{CONCLUSION}

After studying the effect of electrical currents on intergrain barrier ageing, at a fixed (room, in fact) temperature for loosely but dense packing, ${ }^{14-16}$ one step, among many others, consists in studying the temperature dependence of basic transport properties, like the electrical resistivity and the thermoelectric power. One might wonder whether the features are due to the packing structure only or whether the electrothermal properties are sensitive to grain contacts.

We have synthesized a packing of grains made of wellidentified crystalline phases. ${ }^{59}$ The microstructure and chemical content of the tiny grains have been examined. We have measured the electrical resistivity and the thermoelectric power.

The thermoelectric power shows three regimes, like the electrical resistivity - in both cases within identical temperature intervals. Readily, the electrical resistivity has a long unusual power-law decreasing regime between 20 and $200 \mathrm{~K}$. In particular, the thermoelectric power shows an unusual square-root temperature dependence at low temperature. It seems that the observed complex dependencies can be considered to result from a competition between metallic intragrain and semiconducting intergrain barrier properties.

From our observations and reported features by others, we can neglect the inhomogeneity of the grains when discussing electrical and thermal transport properties. The system is thus thought to be described as a network of resistivepacked "macrograins" having boundaries well connected between conducting grains. The role of the density of carriers in the grains is emphasized. Indeed, in view of the rather high value of the overall resistance, even taking into account the effect of macrograin barriers, a sharp DOS at the Fermi level, close to some conducting gap, maybe expected, suggesting some localization. Thus, such electrical and thermal transport properties indicate a competition between mean free paths, later overcome by easy electronic tunneling.

We stress that the corresponding features in the temperature regimes both for $R(T)$ and $Q(T)$ allows for strong arguments on the physics of such packed granular metallic material properties. The $R(T)$ behavior, $\rho \sim T^{-3 / 4}$ at low temperature followed by a linear decrease being unusual for good metallic systems, is interpreted as resulting from a competition process between intragrain and intergrain conductivity. At low temperature, we suggest an intergrain limiting conductivity with intragrain electronic localization and intergrain mediated hopping. An increase in temperature leading to a (low) saturation and delocalization regime.

From the resistance data break, we can even conjecture the existence of a small gap $(\sim 60 \mathrm{~K})$ between the $E_{F}$ level and the top of the valence band in $\mathrm{CaAl}_{2}$, such that thermal excitations reduce the number of truly conducting electrons by exciting them into a narrow (heavy mass) conduction band. This suggests the great interest in calculating the band structure of such compound(s); this could be complemented by specific heat and optical measurements.

Second, it is necessary to insist about the unusual square-root dependence of the thermoelectric power at low temperature, $Q \sim T^{1 / 2}$, and $Q \sim T^{3 / 4}$ at high temperature. Under the conjecture that the behavior is consistent with an electronic hopping between grains at low temperature, we thus report the behavior of a charge carrier on a percolation network under a temperature gradient. A search of the literature has not revealed whether this TEP behaves like that in 
glasses $^{31,32}$ or cermets, nor indicates a behavior controlled by grain-size effects. ${ }^{77}$ Therefore, in agreement with the above considerations on $R(T)$, it may be concluded that such a behavior represents a granular conducting medium thermoelectric power.

An intersting point to be raised here is whether a Peltier effect and a Seebeck effect are equivalent ${ }^{78}$ in granular materials, e.g., would hot spots and aging occur, leading to some hysteresis, if rather than a temperature gradient (Seebeck configuration), a potential difference (Peltier configuration) is applied, and the temperature gradient next measured in order to obtain the TEP characteristics. This is an open question.

In conclusion, there is often some questions about whether single crystals are the only samples of fundamental interest, to be studied and reported upon. No doubt that they easily bring some information on microscopic parameters. Yet, it is also often shown that polycrystalline samples are useful when fine measurements are performed. Moreover, properties of packed systems receive some attention nowadays. To explain their properties become a challenge in condensed matter. In fact, we report an unusual decay of the electrical resistance with temperature, a square root of temperature dependence for the TEP, at low $T$ and some consistent temperature ranges where phenomena can be distinguished due to microscopic processes in metallic grains.

\section{ACKNOWLEDGMENTS}

Work supported in part by the University of Liège Research Council by PST.CLG.977377 and by KBN grant 2PO3B12919, SPUB145, and BE-PL bilateral exchange program.

\section{APPENDIX}

An energy-dispersive X-ray analysis of the synthesized grains indicates that the dendrites (Fig. 1) are Al rich and made of $\mathrm{CaAl}_{2}$, while the matrix is $\mathrm{Al}$ poor and has a composition close to $\mathrm{Ca}_{0.6} \mathrm{Al}_{0.4}$-in fact, a mixture of $\mathrm{Ca}_{13} \mathrm{Al}_{14}$ and $\mathrm{Ca}_{8} \mathrm{Al}_{3}$, taking into account the Huang and Corbett report $^{59}$ and a very recent ${ }^{79}$ phase diagram report sketched in Fig. 6. An x-ray diffraction analysis (Fig. 7) proves that there are in fact three crystalline phases. For identifying the $\mathrm{Ca}_{8} \mathrm{Al}_{13}$ and $\mathrm{Ca}_{13} \mathrm{Al}_{14}$, data from JCPDS files were used. From the $\mathrm{x}$-ray data, the lattice parameter of the $\mathrm{CaAl}_{2}$ phase is found to be $a=0.8045 \mathrm{~nm}$, larger than the one reported as equal to $a=0.8038 \mathrm{~nm}$ in Ref. 58. According to JCPDS 75$0875, a=8.02 \AA$ with $F d-3 m$ as space group. The presence of three phases likely indicates some slight $\mathrm{Ca}$ excess in the initial powder.

A comment is here in order for explaining the growth process-indeed somewhat analogous to the superconducting ceramics mentioned above. Starting from a stoichiometric powder composition close to $\mathrm{CaAl}_{2}$, we consider that the solidification (quenching) process induces the appearance of $\mathrm{CaAl}_{2}$ grains embedded in a fluid phase. Under further cooling, the latter solidifies, $\mathrm{Ca}_{13} \mathrm{Al}_{14}$ precipitates near (or on) $\mathrm{CaAl}_{2}$ grains and decomposes at the $850 \mathrm{~K}$ peritectic temperature into a $\mathrm{Ca}_{13} \mathrm{Al}_{14}$ and a $\mathrm{Ca}_{8} \mathrm{Al}_{3}$ composite matrix leading to the microstructure, schematically described in Fig. 8. In so doing, one can understand that large $\mathrm{CaAl}_{2}$ dendrite grains may be covered by a tiny $\mathrm{Ca}_{13} \mathrm{Al}_{14}$ layer, all embedded in a mainly $\mathrm{Ca}_{0.6} \mathrm{Al}_{0.4}$ matrix containing tiny-less than $1-\mu \mathrm{m}$ size from optical observation, $\mathrm{Ca}_{8} \mathrm{Al}_{3}$ and $\mathrm{Ca}_{13} \mathrm{Al}_{14}$ precipitates. It is interesting to notice that the grains are made of needles in a matrix, in contrast to the rather spherical 211 particles in the 123-YBCO matrix.

${ }^{1}$ G. Deutscher, O. Entin-Wohlman, S. Fishman, and Y. Shapiro, Phys. Rev. B 21, 5041 (1980).

${ }^{2}$ S. Kirkpatrick, Rev. Mod. Phys. 45, 574 (1973).

${ }^{3}$ J. P. Clerc, G. Giraud, S. Alexander, and E. Guyon, Phys. Rev. B 22, 2489 (1980).

${ }^{4}$ H. J. Herrmann, J.-P. Hovi, and S. Luding, Physics of dry granular media (Kluwer, Dordrecht, 1998).

5 J. Duran, Sands, Powders and Grains (Springer, Berlin, 1999).

${ }^{6} \mathrm{~B}$. V. Derjaguin, Theory of stability of colloids and thin films (Consultants Bureau, New York, London, 1989).

${ }^{7}$ A. Gadomski and J. M. Rubí, Chem. Phys. 293, 169 (2003).

${ }^{8}$ H. Hertz, J. Reine, J. Reine Angew. Math. 92, 156 (1895).

${ }^{9}$ A. J. Hunt, T. R. Steyer, and D. R. Huffman, Surf. Sci. 36, 454 (1973).

${ }^{10}$ D. Stroud and F. P. Pan, Phys. Rev. B 17, 1602 (1978).

${ }^{11}$ J. M. Gérardy and M. Ausloos, Phys. Rev. B 25, 4204 (1982).

${ }^{12}$ P. Clippe and M. Ausloos, Phys. Status Solidi B 110, 211 (1982).

${ }^{13}$ I. Runge, Z. Tech. Phys. (Leipzig) 6, 61 (1925).

${ }^{14}$ S. Dorbolo, M. Ausloos, and N. Vandewalle, Appl. Phys. Lett. 81, 936 (2002).

${ }^{15}$ S. Dorbolo, M. Ausloos, and N. Vandewalle, Eur. Phys. J. B 34, 201 (2003).

${ }^{16}$ S. Dorbolo, M. Ausloos, and N. Vandewalle, Phys. Rev. E 67, 040302 (2003).

${ }^{17}$ T. Calzecchi-Onesti, Il Nuovo Cimento 16, 58 (1884).

${ }^{18}$ S. Dorbolo, M. Ausloos, N. Vandewalle, and M. Houssa, J. Appl. Phys. 94, 7835 (2003).

${ }^{19}$ M. Ausloos, Physica B 108, 969 (1981).

${ }^{20}$ B. Abeles, P. Sheng, M. D. Coutts, and Y. Arie, Adv. Phys. 24, 407 (1975).

${ }^{21}$ J. A. A. J. Perenboom, P. Wyder, and F. Meier, Phys. Rep. 78, 173 (1981).

${ }^{22}$ A. I. Voitenko and A. M. Gabovich, Phys. Solid State 41, 1598 (1999).

${ }^{23}$ G. G. N. Angilella, R. Pucci, F. Siringo, and A. Sudbo, in Symmetry and Pairing in Superconductors edited by M. Ausloos and S. Kruchinin (Kluwer Academic Publishers, Dordrecht, 1999).

${ }^{24}$ B. Zeimetz, N. A. Rutter, B. A. Glowacki, and J. E. Evetts, Supercond. Sci. Technol. 14, 672 (2001).

${ }^{25}$ T. C. Choy and A. M. Stoneham, J. Phys.: Condens. Matter 2, 939 (1990).

${ }^{26}$ A. V. Danilov, D. S. Golubev, and S. E. Kubatkin, Phys. Rev. B 65, 125312 (2002).

${ }^{27}$ K. B. Efetov and A. Tschersich, Europhys. Lett. 59, 114 (2002).

${ }^{28}$ I. S. Beloborodov, K. B. Efetov, A. V. Lopatin, and V. M. Vinokur, http://arXiv.org/abs/ cond-mat/0304448; to be published in the Proceedings of NATO Advanced Research Workshop (St. Petersburg, 2003).

${ }^{29}$ M. A. Ryan, R. M. Williams, C. E. Allevato, C. B. Vining, C. K. LoweMa, and S. B. Robie, J. Phys. Chem. Solids 55, 1255 (1994).

${ }^{30}$ C. B. Vining, W. Laskow, J. O. Hanson, R. R. Van der Beck, and P. D. Gorsuch, J. Appl. Phys. 69, 4333 (1991).

${ }^{31}$ N. T. Kemp, A. M. Stewart, J. E. Downes, and A. B. Kaiser, J. Magn. Magn. Mater. 117-118, 129 (1998).

${ }^{32}$ J. R. Cooper, Z. Vucic, and E. Babic, J. Phys. F: Met. Phys. 4, 1489 (1974).

${ }^{33}$ W. A. Phillips, in Topics in Current Physics, Amorphous Solids: Low Temperature Properties Vol. 24, edited by W. A. Phillips (Springer, Berlin, 1981).

${ }^{34}$ R. Zallen, The Physics of Amorphous Solids (Wiley, New York, 1998).

${ }^{35}$ R. Zeller and R. Pohl, Phys. Rev. B 4, 2029 (1971).

${ }^{36}$ N. F. Mott and E. A. Davies, Electron Processes in NonCrystalline Materials (Clarendon, Oxford, 1979).

${ }^{37}$ N. F. Mott, Metal-Insulator Transitions (Taylor \& Francis, London, 1990).

${ }^{38}$ B. I. Shklovskii and A. I. Efros, Electronic Properties of Doped Semiconductors (Springer, Berlin, 1984).

${ }^{39}$ D. Vandembroucq, A. C. Boccara, and S. Roux, J. Phys. III 7, 303 (1997).

${ }^{40}$ R. Piasecki, Phys. Status Solidi B 209, 403 (1998). 
${ }^{41}$ J. M. Ziman, Electrons and Phonons (Clarendon, Oxford, 1962).

${ }^{42}$ K. Durczewski and M. Ausloos, Z. Phys. B: Condens. Matter 85, 59 (1991); (E) 92, 409 (1993); K. Durczewski and M. Ausloos, Phys. Rev. B 49, 13215 (1994);(E) Phys. Rev. B 51, 3320 (1995).

${ }^{43}$ R. A. Roemer, A. MacKinnon, and C. Villagonzalo, J. Phys. Soc. Jpn. 72 Suppl. A, 167 (2003).

${ }^{44}$ D. Stroud, Phys. Rev. B 12, 3368 (1975).

${ }^{45}$ D. A. G. Bruggeman, Ann. Phys. (Leipzig) 24, 636 (1935).

${ }^{46}$ J. Bernasconi and H. J. Wiesmann, Phys. Rev. B 13, 1131 (1976).

${ }^{47}$ M. Hori and F. Yonezawa, J. Phys. C 10, 229 (1977).

${ }^{48}$ M. Nakamura, Phys. Rev. B 28, 2216 (1983).

${ }^{49}$ D. J. Bergman, J. Phys. C 12, 4947 (1977).

${ }^{50}$ A. S. Sangani and A. Acrivos, Proc. R. Soc. London, Ser. A 386, 263 (1983).

${ }^{51}$ L. Poladian and R. C. McPhedran, Proc. R. Soc. London, Ser. A 386, 263 (1983).

${ }^{52}$ A. K. Sen and S. Torquato, Phys. Rev. B 39, 4504 (1989).

${ }^{53}$ A. A. Snarskii and M. Zhenirivskiy, Physica B 322, 84 (2002).

${ }^{54}$ A. S. Skal, Sov. Phys. JETP 61, 302 (1985).

${ }^{55}$ T. K. Xia and X. C. Zeng, J. Phys. C 120, L907 (1987).

${ }^{56}$ D. J. Bergman and D. Stroud, Solid State Physics (Academic, New York, 1992), Vol. 46, p. 147.

${ }^{57}$ D. J. Bergman and L. G. Fel, J. Appl. Phys. 85, 8205 (1999).

${ }^{58}$ T. B. Massalski, Binary Alloy Phase Diagrams, (ASM Intl., Metals Park, Ohio, 1990).

${ }^{59}$ B. Huang and J. D. Corbett, Inorg. Chem. 37, 5827 (1998).

${ }^{60}$ Ch. Laurent, S. K. Patapis, M. Laguesse, H. W. Vanderschueren, A. Rulmont, P. Tarte, and M. Ausloos, Solid State Commun. 66, 445 (1989).

${ }^{61}$ H. Bougrine and M. Ausloos, Rev. Sci. Instrum. 66, 199 (1995).
${ }^{62}$ M. Pȩkala, A. Tampieri, G. Celotti, M. Houssa, and M. Ausloos, Supercond. Sci. Technol. 9, 644 (1996).

${ }^{63}$ A. L. Efros and B. L. Shklovskii, J. Phys. C 8, L49 (1975).

${ }^{64}$ T. Nakayama, K. Yakubo, and R. L. Orbach, Rev. Mod. Phys. 66, 381 (1994).

${ }^{65}$ D. Stauffer and A. Aharony, Introduction to Percolation Theory (Taylor and Francis, London, 1994).

${ }^{66}$ A. V. Danilov, D. S. Golubev, and S. E. Kubatkin, Phys. Rev. B 65 , 125312 (2002)

${ }^{67}$ H. Schweitzer and G. Czycholl, Phys. Rev. Lett. 67, 3724 (1991).

${ }^{68}$ B. Coqblin, in XII School of Modern Physics on Phase Transitions and Critical Phenomena, Làdek Zdrój, Poland in press.

${ }^{69}$ V. L. Ginzburg, Usp. Fiz. Nauk 41, 307 (1998).

${ }^{70}$ P. A. Lee and T. V. Ramakrishnan, Rev. Mod. Phys. 57, 287 (1985).

${ }^{71}$ S. R. Khan, E. M. Pedersen, B. Kain, A. J. Jordan, and R. P. Barber, Jr., Phys. Rev. B 61, 5909 (2000).

${ }^{72}$ A. Gerber, A. Milner, G. Deutscher, M. Karpovsky, and A. Gladkikh, Phys. Rev. Lett. 78, 4277 (1997).

${ }^{73}$ K. Durczewski and M. Ausloos, Phys. Rev. B 53, 1762 (1996);(E) Phys. Rev. B 54, 17224 (1996).

${ }^{74}$ H. P. R. Frederikse, Phys. Rev. 92, 248 (1953).

${ }^{75}$ T. H. Geballe and G. W. Hull, Phys. Rev. 94, 1134 (1954).

${ }^{76}$ C. Herring, Phys. Rev. 96, 1163 (1954).

${ }^{77}$ G. S. Nolas, J. Sharp, and H. J. Goldsmid, Thermoelectrics. Basic Principles and New Materials Developments (Springer, Berlin, 2001).

${ }^{78}$ L. I. Anatychuk, Physics of Thermoelectricity (Inst. Thermoelectricity Kyiv, Chernivtsi, 1998).

${ }^{79}$ K. Ozturk, L.-Q. Chen, and Z.-K. Liu, J. Alloys Compd. 340, 199 (2002). 